\title{
OEstado da Arte da Imunoterapia no Tratamento do Câncer de Mama Triplo-Negativo: Principais Drogas, Associações, Mecanismos de Ação e Perspectivas Futuras
}

doi: https://doi.org/10.32635/2176-9745.RBC.2021v67n2.1014

\author{
The State-of-the-Art of Immunotherapy in the Triple-Negative Breast Cancer Treatment: Main Drugs, Combinations, \\ Mechanisms of Action and Future Perspectives \\ El Estado del Arte de la Inmunoterapia en el Tratamiento del Cáncer de Mama Triple Negativo: Principales Fármacos, \\ Asociaciones, Mecanismos de Acción y Perspectivas de Futuro
}

Dione Fernandes Tavares'; Laércio Moreira Cardoso-Júnior² ${ }^{2}$ Victoria Chaves Ribeiro ${ }^{3}$; Renata Lopes Britto ${ }^{4}$

RESUMO

Introduçáo: $\mathrm{O}$ câncer de mama é o mais comumente diagnosticado em mulheres e uma das principais causas de morte por câncer em mulheres em todo o mundo. Apesar, ou talvez por causa, de sua natureza agressiva e da falta de tratamentos direcionados atuais, pesquisas clínicas e laboratoriais significativas estão fornecendo opçôes de tratamento diferenciadas. Historicamente, a quimioterapia tem sido a única opção viável de tratamento sistêmico para doenças precoces e avançadas. No entanto, ensaios clínicos publicados recentemente mostraram que a imunoterapia tem um papel importante no paradigma de tratamento dessa condição devastadora. Objetivo: Demonstrar o estado da arte da imunoterapia no tratamento do câncer de mama triplo-negativo. Método: Revisão integrativa de literatura, entre janeiro/2020 a março/2020, a partir das bases de dados PubMed, SciELO, International Clinical Trials Registry Platform e LILACS, por meio dos descritores "Imunoterapia", "Neoplasias da mama" e "Neoplasias de mama triplo negativas" e seus respectivos correspondentes em inglês. Resultados: Foram encontrados 465 artigos; destes, 457 foram excluídos após aplicação dos critérios metodológicos. Assim, restaram oito artigos que atendiam aos critérios de inclusão, demonstrando os principais agentes terapêuticos utilizados, mecanismos de ação e combinaçôes terapêuticas. Encontraram-se 25 ensaios clínicos em andamento na plataforma de registro de ensaios clínicos International Clinical Trials Registry Platform. Conclusáo: Embora a imunoterapia seja algo recente, seus resultados com agentes inibidores da PARP, PD-1 e PD-L1 demonstraram resultados satisfatórios. Novos ensaios com subgrupos estratificados de acordo com biomarcadores tumorais específicos são necessários, a fim de avaliar se algum subgrupo tem maior benefício ao tratamento.

Palavras-chave: Imunoterapia; Neoplasias da Mama/terapia; Neoplasias de Mama Triplo Negativas; Biomarcadores.

\section{ABSTRACT}

Introduction: Breast cancer is the most commonly diagnosed cancer in women and is one of the leading causes of death from cancer in women worldwide. Despite, or perhaps because of its aggressive nature and current lack of targeted treatments, significant basic research and clinical trials are being conducted to provide new treatment options. Historically, chemotherapy has been the only viable systemic treatment option for early and advanced diseases. However, recently published clinical trials have shown that immunotherapy plays an important role in the treatment paradigm of this devastating clinical condition. Objective: To demonstrate the state-of-the-art results of immunotherapy in the treatment of triple-negative breast cancer. Method: An integrative literature review was carried out between January/2020 and March/2020, in PubMed, SciELO, International Clinical Trials Registry Platform and LILACS databases, using the keywords "Immunotherapy", "Breast Cancer", and "Triple Negative Breast Cancer" and its respective correspondents in Portuguese. Results: 465 articles were found; of those, 457 were excluded after applying the methodological criteria. Thus, 8 articles that met the inclusion criteria, showing the main therapeutic agents used, mechanisms of action and therapeutic combinations, remained. 25 clinical trials were found in progress on the International Clinical Trials Registry Platform. Conclusion: Although immunotherapy is somewhat recent, its results with PARP, PD-1 and PD-L1 inhibitors have shown satisfactory results. New trials with subgroups stratified according to specific tumor biomarkers are needed in order to assess if some subgroups have greater benefit to treatment. Key words: Immunotherapy; Breast Neoplasms/therapy; Triple Negative Breast Neoplasms; Biomarkers.

\section{RESUMEN}

Introducción: El cáncer de mama es el más comúnmente diagnosticado en las mujeres y es una de las principales causas de muerte por cáncer en mujeres de todo el mundo. A pesar de, o quizás debido a su naturaleza agresiva y la falta de tratamientos dirigidos actuales, investigaciones clínicas y de laboratorio significativas están proporcionando opciones de tratamiento diferenciadas. Históricamente, la quimioterapia ha sido la única opción viable para el tratamiento sistémico de enfermedades tempranas y avanzadas. Sin embargo, los ensayos clínicos publicados recientemente han demostrado que la inmunoterapia desempeña un papel importante en el paradigma del tratamiento de esta condición devastadora. Objetivo: Demostrar el estado del arte de la inmunoterapia en el cáncer de mama triple negativo. Método: Revisión integradora entre enero/2020 y marzo/2020, utilizando las bases de datos PubMed, SciELO, International Clinical Trials Registry Platform y LILACS, empleando las palabras clave "Inmunoterapia", "Cáncer de mama" y "Cáncer de mama triple negativo" y los respectivos términos en inglés. Resultados: Se encontraron 465 artículos; de estos, 457 fueron excluidos después de aplicar los criterios metodológicos. Así, quedaron 8 artículos que cumplían los criterios, que mostraban los principales agentes terapéuticos utilizados, mecanismos de acción y combinaciones terapéuticas. Se encontraron 25 ensayos clínicos en progreso en la plataforma International Clinical Trials Registry Platform. Conclusión: Aunque la inmunoterapia es algo reciente, sus resultados con inhibidores de PARP, PD-1 y PD-L1 han mostrado resultados satisfactorios. Se necesitan nuevos ensayos con subgrupos estratificados según biomarcadores tumorales específicos para evaluar si algún subgrupo tiene un mayor beneficio.

Palabras clave: Inmunoterapia; Neoplasias de la Mama/terapia; Neoplasias de la Mama Triple Negativas; Biomarcadores.

\footnotetext{
1,2,4 Universidade Federal da Bahia. Faculdade de Medicina da Bahia. Programa de Pós-Graduação em Medicina e Saúde. Salvador (BA), Brasil. E-mails: dionefernandestavares@gmail.com; laerciop53@gmail.com; renatalopesbritto@gmail.com.

Orcid iD: https://orcid.org/0000-0002-2438-5297; Orcid iD: https://orcid.org/0000-0003-0649-1237; Orcid iD: https://orcid.org/0000-0002-8852-7039

3Universidade de São Paulo. Instituto de Química. São Paulo (SP), Brasil. E-mail: victoriaribeiro@usp.br. Orcid iD: https://orcid.org/0000-0003-4612-3009

Endereço para correspondência: Dione Fernandes Tavares. Programa de Pós-Graduação em Medicina e Saúde, Hospital Universitário Professor Edgard Santos. Rua Doutor Augusto Viana, S/No - Canela. Salvador (BA), Brasil. CEP 40110-060. E-mail: dionefernandestavares@gmail.com
} 


\section{INTRODUÇÃO}

O câncer de mama consiste em uma patologia com conhecida variabilidade nas apresentações clínicas, comportando-se de forma altamente heterogênea, composta por diferentes subtipos moleculares. Nesse contexto, denomina-se "triplo-negativo" o tumor que não apresenta nenhum dos três biomarcadores mais empregados na classificação do câncer de mama. São eles: receptor de estrógeno (ER), receptor de progesterona (PR) e proteína HER-2 (HER2) $)^{1,2}$.

O câncer de mama é o tipo mais comumente diagnosticado em mulheres e uma das principais causas de morte por câncer em mulheres em todo o mundo. No Brasil, à exceção do câncer de pele não melanoma, corresponde a cerca de $29,7 \%$ dos casos novos a cada ano $^{3}$. A estimativa de novos casos, para o ano de 2020, no Brasil, foi de 66.280 casos novos, o que equivale à taxa de altíssima incidência de 61,61 casos por 100 mil mulheres $^{3}$. Assim como em outros tipos de câncer, o diagnóstico precoce aumenta muito as chances de sucesso do tratamento, permitindo uma redução de $20 \%$ nas taxas gerais de mortalidade ${ }^{4}$.

Apesar do benefício significativo do uso de quimioterapia convencional e da utilização de anticorpos monoclonais no prognóstico de pacientes com câncer de mama, o tratamento do câncer de mama triplo-negativo ainda é um grande desafio. À vista disso, o tratamento imunológico de tumores de mama encontra-se em pleno desenvolvimento nos último dez anos, com a realização de diversas pesquisas utilizando inibidores da poli (adenosina difosfato-ribose) polimerase (PARP) e inibidores do controle imunológico. A recente aprovação do anticorpo antiligante de morte celular programada 1 (anti-PD-L1) atezolizumab em combinação com quimioterapia foi um marco no tratamento de pacientes com câncer de mama triplo-negativo $^{5-12}$.

Dessa forma, o objetivo deste trabalho é demonstrar o atual estado da arte das pesquisas em imunoterapia no tratamento do câncer de mama triplo-negativo, investigando os principais fármacos, associaçôes, mecanismos de ação e perspectivas futuras em pacientes com câncer de mama triplo-negativo, bem como diferentes abordagens que podem ser úteis para melhorar a resposta dos tumores às imunoterapias.

\section{MÉTODO}

O presente artigo relata uma revisão de literatura, do tipo integrativa, baseada nas recomendaçóes para elaboraçáo desse tipo de revisão ${ }^{13}$, cujo levantamento dos estudos sobre a utilização da imunoterapia no tratamento do câncer de mama triplo-negativo foi realizado a partir da elaboraçáo da pergunta norteadora: "Qual o estado da arte da imunoterapia no tratamento do câncer de mama triplo-negativo?”. Em primeiro lugar, realizou-se o dimensionamento do número de trabalhos indexados em diferentes bases de dados, entre as quais, foram consultadas: a) Scientific Electronic Library Online (SciELO); b) Literatura Latino-Americana e do Caribe em Ciências da Saúde (LILACS); c) U. S. National Library of Medicine (PubMed). A consulta foi realizada de janeiro de 2020 a março de 2020.

Após consulta aos Descritores em Ciências da Saúde (DeCS), elegeram-se os seguintes descritores: "Imunoterapia", "Neoplasias da mama" e "Neoplasias de mama triplo negativas" para realização da busca nas bases de dados SciELO e LILACS. Após consulta aos Medical Subject Headings (MeSH), escolheram-se os seguintes descritores: "Immunotherapy", "Breast Cancer", "Triple Negative Breast Cancer" e "TNBC" para realização da busca na base de dados PubMed e na Plataforma de registro de ensaios clínicos International Clinical Trials Registry Platform, a fim de levantar possíveis futuros ensaios clínicos, utilizando imunoterapia no tratamento do câncer de mama triplo-negativo, descrever a fase em que se encontravam e as perspectivas futuras.

Os critérios de inclusão foram artigos de março de 2010 a março de 2020; em português ou inglês; e o desenho dos estudos deveria corresponder a estudos clínicos, independente da metodologia, com utilização de imunoterapia em pacientes com idade igual ou superior a 18 anos; com diagnóstico de câncer de mama triplo-negativo com confirmação anatomopatológica e por imuno-histoquímica. Excluíram-se da análise qualitativa artigos de opiniâo, editoriais, relatos de casos e/ou experiência, cartas ao editor e comentários. A partir da seleção final dos artigos, foram extraídas as seguintes informações: classificação do tipo de câncer, fármacos utilizados, fase clínica, associaçóes entre imunoterapia e outras terapias já existentes, mecanismo de ação e perspectivas futuras. Foram extraídas também da plataforma de registro de ensaios clínicos International Clinical Trials Registry Platform as seguintes informaçôes dos estudos clínicos em andamento: número de registro, configuração, fase do estudo, fármacos utilizados, número de participantes, instituição realizadora da pesquisa, país e status do andamento do trabalho.

\section{RESULTADOS}

Em virtude da inexistência de trabalhos nas bases de dados SciELO e LILACS, utilizaram-se neste artigo apenas trabalhos da base de dados PubMed. Com base 
nas estratégias definidas para a busca, encontrou-se inicialmente um total de 465 publicaçóes. Depois de removidas as duplicatas $(n=69), 396$ trabalhos seguiram para etapa de triagem com leitura dos títulos e resumos, dos quais, 347 foram excluídos pelos revisores. Os 29 artigos remanescentes foram lidos na íntegra, para aplicação dos critérios de elegibilidade, possibilitando a inclusão final de oito estudos que cumpriam os critérios de inclusão. A estratégia de busca detalhada pode ser observada na Figura 1. No processo de busca, é importante salientar que foram encontradas apenas três revisóes sobre o assunto, sendo que nenhuma delas contemplava o trabalho realizado neste artigo.

Após a realização dos processos metodológicos de identificação, triagem e elegibilidade dos estudos (Figura 1), pôde-se estratificar e reunir as principais informações sobre a classificação do câncer, assim como seu estágio clínico atual, o fármaco imunoterápico e seu mecanismo de ação no sistema imunológico, além da sua possível associação com agentes quimioterápicos já utilizados amplamente. Os dados estratificados dos artigos incluídos na síntese qualitativa podem ser observados no Quadro 1.

Em relação às perspectivas futuras da utilização da imunoterapia no câncer de mama triplo-negativo, realizou-se uma busca de ensaios clínicos em andamento na plataforma ClinicalTrials.gov, mantida pelo PubMed, que contém registros de estudos de 210 diferentes países.
No Quadro 2, observam-se diferentes pesquisas realizadas em diferentes regióes, utilizando imunoterapia no tratamento do câncer de mama triplo-negativo.

\section{DISCUSSÃO}

A proteína programada para morte celular 1 (PD-1) é uma proteína reguladora encontrada na superfície de células efetoras do sistema imune, as células $\mathrm{T}$, e possui ação inibitória. Em condições fisiológicas, a interação desta com seu ligante (PD-L1) promove a regulação da reaçáo imunológica, diminuindo a ação efetora do sistema imune. Dessa forma, quando expresso na superfície de células tumorais, o ligante PD-L1 permite a evasão da resposta imune pelo tumor ${ }^{13}$. Com o avançar do conhecimento científico, o papel da interação da PD-1 com seu receptor-ligante PD-L1 foi destacado como importante via inibidora, que pode ser sequestrada por tumores para suprimir o controle imunológico ${ }^{13}$.

Quando os ligantes de PD-1 se juntam à PD-1, a ativação de células $T$ por meio do receptor de células $T$ é inibida. O PD-L1, que é o ligante PD-1, é predominantemente envolvido na regulação negativa da função das células $T$ no tecido periférico e pode ser expresso em vários tipos de câncer, por isso a aplicação de agentes inibidores de PD-1/PD-L1 também é estudada em outros tipos de câncer com resultados promissores, como no caso do

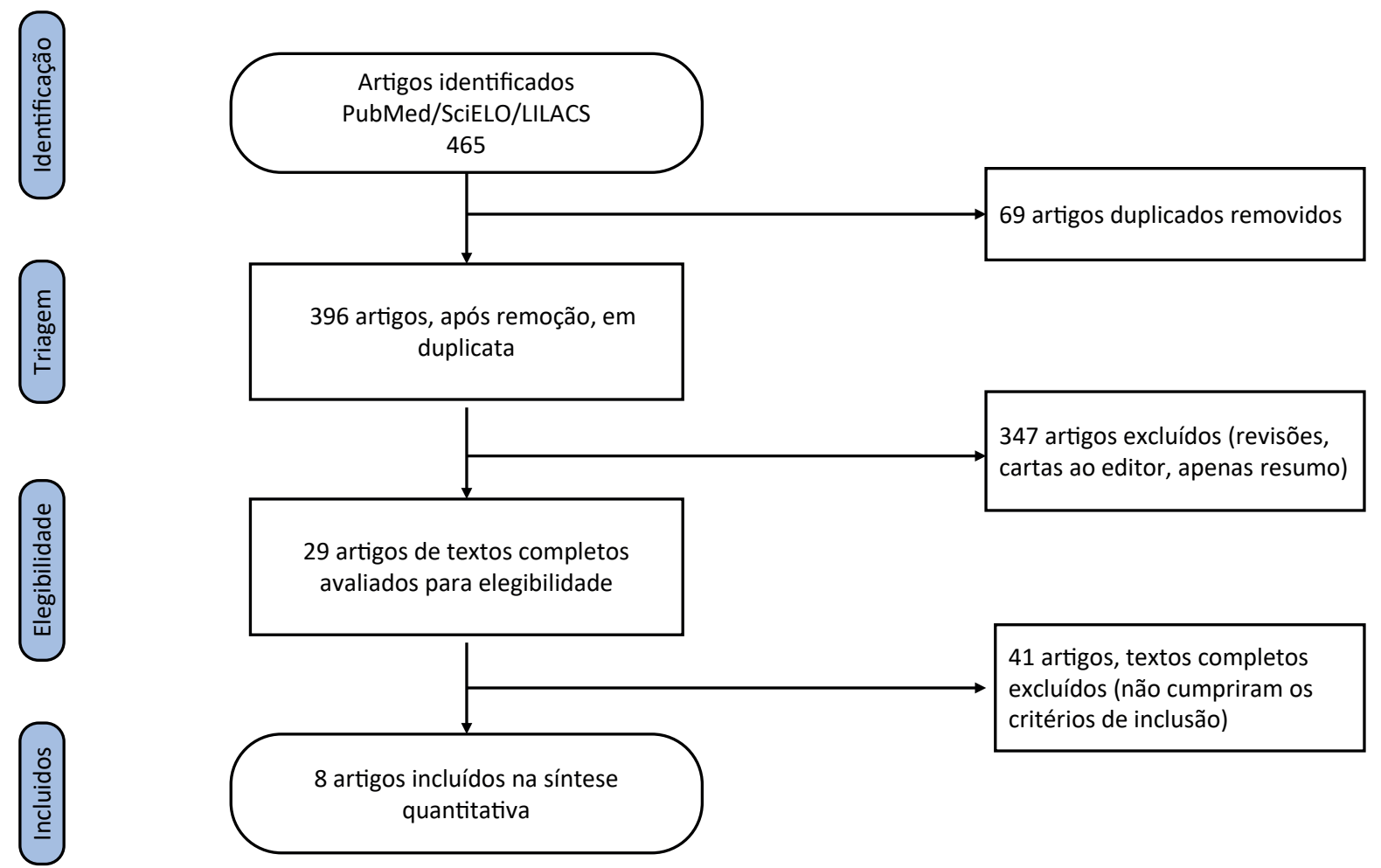

Figura 1. Fluxograma do processo de seleção dos artigos nas diferentes fases da revisão 
Quadro 1. Principais fármacos, associações e mecanismos de ação de imunoterapia no tratamento do câncer de mama triplo-negativo descritos em ensaios clínicos

\begin{tabular}{|c|c|c|c|c|c|}
\hline Autor/ano & $\mathbf{N}$ & $\begin{array}{l}\text { Classificação do } \\
\text { câncer }\end{array}$ & Fármaco & Associação & $\begin{array}{c}\text { Mecanismo de } \\
\text { ação }\end{array}$ \\
\hline $\begin{array}{l}\text { Schmid et al., } \\
2018^{5}\end{array}$ & 902 & $\begin{array}{c}\text { Câncer de mama } \\
\text { triplo-negativo } \\
\text { avançado metastático }\end{array}$ & Atezolizumab & Nab-paclitaxel & Inibidor PD-L1 \\
\hline $\begin{array}{l}\text { Emens et al., } \\
2019^{10}\end{array}$ & 116 & $\begin{array}{l}\text { Câncer de mama } \\
\text { triplo-negativo } \\
\text { avançado }\end{array}$ & Atezolizumab & Monoterapia & Inibidor PD-L1 \\
\hline $\begin{array}{c}\text { Dirix et al., } \\
2018^{9}\end{array}$ & 58 & $\begin{array}{l}\text { Câncer de mama } \\
\text { triplo-negativo }\end{array}$ & Avelumab & Monoterapia & Inibidor PD-L1 \\
\hline $\begin{array}{l}\text { Nanda et al., } \\
2016^{6}\end{array}$ & 32 & $\begin{array}{c}\text { Câncer de mama } \\
\text { triplo-negativo } \\
\text { avançado }\end{array}$ & Pembrolizumab & Monoterapia & Inibidor PD-1 \\
\hline $\begin{array}{l}\text { Adams et al., } \\
2019^{7}\end{array}$ & 170 & $\begin{array}{l}\text { Câncer de mama } \\
\text { triplo-negativo } \\
\text { avançado e não } \\
\text { tratável }\end{array}$ & Pembrolizumab & Monoterapia & Inibidor PD-1 \\
\hline $\begin{array}{l}\text { Tolaney et al., } \\
2018^{8}\end{array}$ & 107 & $\begin{array}{l}\text { Câncer de mama } \\
\text { triplo-negativo } \\
\text { avançado }\end{array}$ & Pembrolizumab & Eribulina & $\begin{array}{l}\text { Inibidor PD-1 + } \\
\text { quimioterapia }\end{array}$ \\
\hline $\begin{array}{l}\text { Loibl et al., } \\
2018^{11}\end{array}$ & 316 & $\begin{array}{l}\text { Câncer de mama } \\
\text { triplo-negativo }\end{array}$ & Veliparib & Carboplatina & $\begin{array}{c}\text { Inibidor da PARP + } \\
\text { quimioterapia }\end{array}$ \\
\hline $\begin{array}{l}\text { Litton et al., } \\
2018^{12}\end{array}$ & 15 & $\begin{array}{c}\text { Câncer de mama } \\
\text { triplo-negativo } \\
\text { operável com variante } \\
\text { patogênica de } \\
\text { linhagem germinativa } \\
\text { em BRCA }\end{array}$ & Talazoparib & $\begin{array}{l}\text { Monoterapia } \\
+ \text { ressecção } \\
\text { cirúrgica }\end{array}$ & Inibidor da PARP \\
\hline
\end{tabular}

Legendas: PD-1 = Proteína programada para morte celular 1; PD-L1 = Ligante da proteína programada para morte celular 1; PARP = poli (adenosina difosfatoribose) polimerase.

melanoma. Consequentemente, a interrupçáo desse sistema regulador tornou-se um dos alvos terapêuticos mais atraentes na imunoterapia de câncer nos últimos dez anos, conforme demonstrado na maioria dos ensaios clínicos aqui retratados (Quadro 1), correspondente a seis dos oito estudos aqui elencados ${ }^{13,14}$.

O atezolizumab é um anticorpo monoclonal já utilizado no tratamento dos cânceres de pulmáo e bexiga. Seu mecanismo de atuação consiste na inibição do PD-L1. Foram encontrados dois ensaios clínicos com o fármaco. No primeiro, o atezolizumab foi associado a um taxano, o nab-paclitaxel, cujos resultados demonstraram um prolongamento da sobrevida livre de progressão do câncer de mama triplo-negativo metastático na população tratada e no subgrupo no qual o PD-L1 foi positivo na avaliação imuno-histoquímica. Além disso, os eventos adversos foram consistentes com os perfis de segurança conhecidos de cada droga, demonstrando segurança na utilização ${ }^{5}$. O segundo estudo traz a utilização do atezolizumab também em indivíduos com câncer de mama triplo-negativo metastático que já tentaram uma ou mais linhas de tratamento anteriormente sem sucesso. Dessa vez utilizado como monoterapia, observou-se, em dois anos de acompanhamento, uma resposta clínica durável. Os pacientes com maior infiltração de células imunes ao tumor, coradas por imuno-histoquímica, tiveram melhores resultados clínicos ${ }^{10}$.

Outro inibidor do PD-L1 também foi encontrado em um dos oito artigos selecionados (Quadro 1). O avelumab foi utilizado em pacientes com câncer de mama local avançado ou metastático, que já tinham se submetido a três ou mais linhas de tratamento previamente sem sucesso. Por se tratar de um estudo inicial, pretendia avaliar a segurança do uso da droga e obteve uma resposta aceitável à sua utilização. Houve uma taxa de resposta significativamente melhor no grupo que apresentava expressáo de PD-L1 por imuno-histoquímica, levando à hipótese de que a expressão de PD-L1 em células imunes 
Quadro 2. Ensaios clínicos em andamento, utilizando imunoterapia no tratamento do câncer de mama triplo-negativo

\begin{tabular}{|c|c|c|c|c|c|c|}
\hline Registro & Configuração & Fase & Fármacos & $\mathbf{N}$ & Instituição (país) & Status \\
\hline NCT03036488 & $\begin{array}{l}\text { Neoadjuvante / } \\
\text { Adjuvante }\end{array}$ & III & $\begin{array}{l}\text { Pembrolizumab } \\
\text { e quimioterapia } \\
\text { versus placebo e } \\
\text { quimioterapia como } \\
\text { terapia neoadjuvante e } \\
\text { pembrolizumab versus } \\
\text { placebo como terapia } \\
\text { adjuvante }\end{array}$ & 1.174 & $\begin{array}{c}\text { Merck Sharp \& } \\
\text { Dohme Corp. (US) }\end{array}$ & $\begin{array}{l}\text { Ativo, sem } \\
\text { recrutamento } \\
\text { no momento }\end{array}$ \\
\hline NCT03281954 & $\begin{array}{c}\text { Neoadjuvante/ } \\
\text { Adjuvante }\end{array}$ & III & $\begin{array}{c}\text { Quimioterapia e } \\
\text { atezolizumab ou } \\
\text { placebo seguido por } \\
\text { continuação adjuvante } \\
\text { de atezolizumab ou } \\
\text { placebo }\end{array}$ & 1.520 & $\begin{array}{c}\text { Nsabp Foundation } \\
\text { Inc (US) }\end{array}$ & Recrutamento \\
\hline NCT03197935 & $\begin{array}{l}\text { Neoadjuvante/ } \\
\text { Adjuvante }\end{array}$ & III & $\begin{array}{l}\text { Atezolizumab e } \\
\text { quimioterapia } \\
\text { neoadjuvante à base } \\
\text { de antraciclina/ } \\
\text { nab-paclitaxel } \\
\text { em comparação } \\
\text { com placebo e } \\
\text { quimioterapia }\end{array}$ & 204 & $\begin{array}{l}\text { Hoffmann-La } \\
\text { Roche (CH) }\end{array}$ & $\begin{array}{l}\text { Ativo, sem } \\
\text { recrutamento } \\
\text { no momento }\end{array}$ \\
\hline NCT03756298 & $\begin{array}{l}\text { Adjuvante } \\
\text { apenas para } \\
\text { pacientes } \\
\text { com doença } \\
\text { residual após } \\
\text { quimioterapia } \\
\text { neoadjuvante }\end{array}$ & II & $\begin{array}{l}\text { Atezolizumab mais } \\
\text { terapia adjuvante } \\
\text { com capecitabina } \\
\text { em comparação à } \\
\text { monoterapia com } \\
\text { capecitabina }\end{array}$ & 284 & $\begin{array}{l}\text { National Cancer } \\
\text { Center (KOR) }\end{array}$ & Recrutamento \\
\hline NCT03498716 & Adjuvante & III & $\begin{array}{c}\text { Atezolizumab e } \\
\text { quimioterapia à base } \\
\text { de antraciclina/taxano } \\
\text { adjuvante versus } \\
\text { quimioterapia isolada }\end{array}$ & 2.300 & $\begin{array}{l}\text { Hoffmann-La } \\
\text { Roche }(\mathrm{CH})\end{array}$ & Recrutamento \\
\hline NCT03125902 & $\begin{array}{l}\text { Câncer de mama } \\
\text { triplo-negativo } \\
\text { avançado local } \\
\text { ou metastático }\end{array}$ & III & $\begin{array}{c}\text { Atezolizumab em } \\
\text { combinação com } \\
\text { paclitaxel comparado } \\
\text { com placebo associado } \\
\text { a paclitaxel }\end{array}$ & 540 & $\begin{array}{l}\text { Hoffmann-La } \\
\text { Roche }(\mathrm{CH})\end{array}$ & Recrutamento \\
\hline NCT03164993 & $\begin{array}{l}\text { Câncer de mama } \\
\text { triplo-negativo } \\
\text { avançado local } \\
\text { ou metastático }\end{array}$ & II & $\begin{array}{l}\text { Atezolizumab } \\
\text { combinado com } \\
\text { quimioterapia } \\
\text { imunogênica }\end{array}$ & 75 & $\begin{array}{l}\text { Oslo University } \\
\text { Hospital (NO) }\end{array}$ & Recrutamento \\
\hline NCT03206203 & $\begin{array}{l}\text { Câncer de mama } \\
\text { triplo-negativo } \\
\text { avançado local } \\
\text { ou metastático }\end{array}$ & II & $\begin{array}{l}\text { Carboplatina com ou } \\
\text { sem atezolizumab }\end{array}$ & 185 & $\begin{array}{l}\text { Vanderbilt-Ingram } \\
\text { Cancer Center } \\
\text { (US) }\end{array}$ & Recrutamento \\
\hline NCT03371017 & $\begin{array}{l}\text { Câncer de mama } \\
\text { triplo-negativo } \\
\text { avançado local } \\
\text { ou metastático }\end{array}$ & III & $\begin{array}{l}\text { Atezolizumab e } \\
\text { quimioterapia }\end{array}$ & 350 & $\begin{array}{l}\text { Hoffmann-La } \\
\text { Roche (CH) }\end{array}$ & Recrutamento \\
\hline NCT02926196 & Adjuvante & III & $\begin{array}{l}\text { Avelumab como } \\
\text { tratamento adjuvante } \\
\text { ou pós-tratamento } \\
\text { neoadjuvante }\end{array}$ & 335 & $\begin{array}{l}\text { Istituto Oncologico } \\
\text { Veneto IRCCS (IT) }\end{array}$ & Recrutamento \\
\hline
\end{tabular}


Quadro 2. continuação

\begin{tabular}{|c|c|c|c|c|c|c|}
\hline Registro & Configuração & Fase & Fármacos & $\mathbf{N}$ & Instituição (país) & Status \\
\hline NCT03639948 & Neoadjuvante & II & $\begin{array}{l}\text { Pembrolizumab e } \\
\text { carboplatina mais } \\
\text { docetaxel }\end{array}$ & 100 & $\begin{array}{l}\text { University of } \\
\text { Kansas Medical } \\
\text { Center (US) }\end{array}$ & Recrutamento \\
\hline NCT03289819 & Neoadjuvante & II & $\begin{array}{l}\text { Pembrolizumab } \\
\text { neoadjuvante em } \\
\text { combinação com nab- } \\
\text { - paclitaxel seguido } \\
\text { por pembrolizumab } \\
\text { em combinação } \\
\text { com epirrubicina e } \\
\text { ciclofosfamida }\end{array}$ & 50 & $\begin{array}{l}\text { Institut fuer } \\
\text { Frauengesund heit } \\
\text { (GER) }\end{array}$ & Recrutamento \\
\hline NCT02954874 & $\begin{array}{l}\text { Adjuvante } \\
\text { apenas para } \\
\text { pacientes } \\
\text { com doença } \\
\text { residual após } \\
\text { quimioterapia } \\
\text { neoadjuvante }\end{array}$ & III & $\begin{array}{l}\text { Pembrolizumab como } \\
\text { terapia adjuvante }\end{array}$ & 1.000 & $\begin{array}{l}\text { National Cancer } \\
\text { Institute (US) }\end{array}$ & Recrutamento \\
\hline NCT02768701 & $\begin{array}{l}\text { Câncer de mama } \\
\text { triplo-negativo } \\
\text { avançado local } \\
\text { ou metastático }\end{array}$ & II & $\begin{array}{c}\text { Ciclofosfamida em } \\
\text { dose única associada a } \\
\text { pembrolizumab }\end{array}$ & 40 & $\begin{array}{l}\text { UNC Lineberger } \\
\text { Comprehensive } \\
\text { Cancer Center } \\
\text { (US) }\end{array}$ & $\begin{array}{l}\text { Ativo, sem } \\
\text { recrutamento } \\
\text { no momento }\end{array}$ \\
\hline NCT03121352 & $\begin{array}{l}\text { Câncer de mama } \\
\text { triplo-negativo } \\
\text { avançado local } \\
\text { ou metastático }\end{array}$ & II & $\begin{array}{l}\text { Carboplatina, } \\
\text { nab-paclitaxel e } \\
\text { pembrolizumab }\end{array}$ & 30 & $\begin{array}{c}\text { Case } \\
\text { Comprehensive } \\
\text { Cancer Center } \\
\text { (US) }\end{array}$ & Recrutamento \\
\hline NCT02819518 & $\begin{array}{l}\text { Câncer de mama } \\
\text { triplo-negativo } \\
\text { avançado local } \\
\text { ou metastático }\end{array}$ & III & $\begin{array}{l}\text { Pembrolizumab } \\
\text { mais quimioterapia } \\
\text { versus placebo mais } \\
\text { quimioterapia }\end{array}$ & 858 & $\begin{array}{c}\text { Merck Sharp \& } \\
\text { Dohme Corp. (US) }\end{array}$ & $\begin{array}{l}\text { Ativo, sem } \\
\text { recrutamento } \\
\text { no momento }\end{array}$ \\
\hline NCT02555657 & $\begin{array}{l}\text { Câncer de mama } \\
\text { triplo-negativo } \\
\text { avançado local } \\
\text { ou metastático }\end{array}$ & III & $\begin{array}{c}\text { Pembrolizumab versus } \\
\text { quimioterapia de } \\
\text { agente único }\end{array}$ & 600 & $\begin{array}{c}\text { Merck Sharp \& } \\
\text { Dohme Corp. (US) }\end{array}$ & $\begin{array}{l}\text { Ativo, sem } \\
\text { recrutamento } \\
\text { no momento }\end{array}$ \\
\hline NCT03644589 & $\begin{array}{l}\text { Câncer de mama } \\
\text { triplo-negativo } \\
\text { avançado local } \\
\text { ou metastático }\end{array}$ & II & $\begin{array}{l}\text { Pembrolizumab e } \\
\text { cisplatina }\end{array}$ & 60 & $\begin{array}{c}\text { Merck Sharp \& } \\
\text { Dohme Corp. (US) }\end{array}$ & $\begin{array}{l}\text { Não recrutado } \\
\text { Terapia de } \\
\text { escolha } \\
\text { médica }\end{array}$ \\
\hline NCT02755272 & $\begin{array}{l}\text { Câncer de mama } \\
\text { triplo-negativo } \\
\text { avançado local } \\
\text { ou metastático }\end{array}$ & II & $\begin{array}{c}\text { Pembrolizumab } \\
\text { com carboplatina e } \\
\text { gemcitabina }\end{array}$ & 87 & $\begin{array}{l}\text { Fox Chase Cancer } \\
\text { Center (US) }\end{array}$ & Recrutamento \\
\hline NCT02447003 & $\begin{array}{l}\text { Câncer de mama } \\
\text { triplo-negativo } \\
\text { avançado local } \\
\text { ou metastático }\end{array}$ & II & $\begin{array}{l}\text { Monoterapia com } \\
\text { pembrolizumab }\end{array}$ & 285 & $\begin{array}{c}\text { Merck Sharp \& } \\
\text { Dohme Corp. (US) }\end{array}$ & $\begin{array}{l}\text { Ativo, sem } \\
\text { recrutamento } \\
\text { no momento }\end{array}$ \\
\hline NCT03356860 & Neoadjuvante & II & $\begin{array}{l}\text { Durvalumab } \\
\text { combinado com } \\
\text { quimioterapia } \\
\text { neoadjuvante }\end{array}$ & 57 & $\begin{array}{l}\text { Grand Hôpital de } \\
\text { Charleroi (BE) }\end{array}$ & Recrutamento \\
\hline NCT02685059 & Neoadjuvante & II & $\begin{array}{l}\text { Durvalumab e } \\
\text { quimioterapia com } \\
\text { taxano/antraciclina }\end{array}$ & 174 & $\begin{array}{c}\text { German Breast } \\
\text { Group (GE) }\end{array}$ & $\begin{array}{l}\text { Ativo, sem } \\
\text { recrutamento } \\
\text { no momento }\end{array}$ \\
\hline
\end{tabular}


Quadro 2. continuação

\begin{tabular}{|l|c|c|c|c|c|c|}
\hline \multicolumn{1}{|c|}{ Registro } & Configuração & Fase & Fármacos & N & Instituição (país) & Status \\
\hline NCT03606967 & $\begin{array}{l}\text { Câncer de mama } \\
\text { triplo-negativo } \\
\text { avançado local } \\
\text { ou metastático }\end{array}$ & II & $\begin{array}{c}\text { Nab-paclitaxel e } \\
\text { durvalumabe com } \\
\text { ou sem vacina de } \\
\text { neoantígenos }\end{array}$ & 70 & $\begin{array}{c}\text { National Cancer } \\
\text { Institute (US) }\end{array}$ & $\begin{array}{c}\text { Não recrutado } \\
\text { Terapia de } \\
\text { escolha } \\
\text { médica }\end{array}$ \\
\hline NCT03616886 & $\begin{array}{l}\text { Câncer de mama } \\
\text { triplo-negativo } \\
\text { avançado local } \\
\text { ou metastático }\end{array}$ & II & $\begin{array}{c}\text { Paclitaxel em } \\
\text { associação com } \\
\text { carboplatina e } \\
\text { durvalumab com ou } \\
\text { sem oleclumab }\end{array}$ & 171 & $\begin{array}{c}\text { Jules Bordet } \\
\text { Institute (BE) }\end{array}$ & Recrutamento \\
\hline NCT03167619 & $\begin{array}{l}\text { Câncer de mama } \\
\text { triplo-negativo } \\
\text { avançado local } \\
\text { ou metastático }\end{array}$ & II & $\begin{array}{l}\text { Durvalumab e olaparib } \\
\text { em indivíduos tratados } \\
\text { com quimioterapia à } \\
\text { base de platina }\end{array}$ & 60 & Duke University \\
(US)
\end{tabular}

associadas a tumores pode estar relacionada a uma maior probabilidade de resposta clínica ao avelumab?

Além dos inibidores do PD-L1, há também os inibidores da PD-1, aqui representados pelo anticorpo monoclonal pembrolizumab, que foi estudado em três dos oito artigos incluídos neste trabalho (Quadro 1). No primeiro trabalho, o pembrolizumab foi utilizado como monoterapia em pacientes com câncer avançado, no qual as evidências preliminares da atividade clínica e o perfil de segurança foram avaliados. Concluiu-se que a droga é potencialmente aceitável, embora tenha sido descrita uma alta taxa de eventos adversos. Acredita-se que estes estejam relacionados à dose administrada ${ }^{6}$. No segundo ensaio clínico, também foi utilizada a monoterapia em pacientes com câncer avançado, que já haviam realizado tratamento prévio sem sucesso; neste caso, três ou mais outras linhas de tratamento falhas. A monoterapia mostrou atividade antitumoral durável em um pequeno subconjunto de pacientes pré-tratados e demonstrou um perfil de segurança gerenciável, como a maioria dos efeitos adversos classificados como baixo grau $^{7}$. No último trabalho utilizando pembrolizumab, foi realizada uma combinação com eribulina, tratando-se de um estudo inicial para avaliação da segurança, com resultados positivos. Os efeitos adversos foram comparáveis aos observados com o tratamento, utilizando um dos fármacos, como a monoterapia ${ }^{8}$.

Diversas combinações de diferentes princípios ativos vêm sendo utilizadas nos estudos clínicos já realizados (Quadro 1) e nos ensaios clínicos em andamento (Quadro 2). A quimioterapia, por exemplo, pode induzir a múltiplas alteraçóes imunomodulatórias no microambiente tumoral, incluindo o aumento da liberação de antígeno pelas células tumorais, regulação positiva de PD-L1 e hiperexpressão de marcadores imunogênicos da superfície celular (por exemplo, MHC classe I) ${ }^{15,16}$. Coletivamente, essas modificaçôes podem influenciar positivamente a eficácia da imunoterapia, justificando o fato da associação com outros fármacos como a eribulina.

As antraciclinas, aqui exemplificadas pela doxorrubicina e epirrubicina (Quadros 1 e 2), são capazes de induzir a morte celular imunogênica, uma forma de apoptose que pode levar a uma resposta imune antitumoral eficaz, por intermédio da ativação de células dendríticas e resposta específica de células. Além disso, as antraciclinas também podem aumentar a proliferação de células T $\mathrm{CD} 8+{ }^{17}$.

A associação de imunoterápicos com taxanos também é comum nos ensaios clínicos já realizados e em andamento. Acredita-se que os taxanos diminuem seletivamente os linfócitos T-supressores, reduzindo parcialmente a imunossupressão no microambiente tumoral. Esse efeito imunomodulador foi relatado em taxanos da geração antiga (docetaxel e paclitaxel); nenhum dado pré-clínico sobre a atividade do nab-paclitaxel no sistema imunológico foi descrito até o momento ${ }^{18}$. O nab-paclitaxel foi selecionado inicialmente no estudo IMpassion130, uma vez que facilita o uso reduzido de corticosteroides ${ }^{5}$.

Os agentes alquilantes, como a carboplatina, impedem a célula de se reproduzir danificando seu DNA, agindo em todas as fases do ciclo celular, além de outras funçôes específicas como a ciclofosfamida que, além da sua capacidade de induzir a morte celular imunogênica, pode suprimir células reguladoras $\mathrm{T}$ e aumentar a capacidade proliferativa de células T CD8 + e células $\mathrm{NK}^{19}$.

Por fim, os inibidores da PARP foram estudados em dois dos oito artigos incluídos (Quadro 1), fármacos aqui representados pelo veliparib e talazoparib. As PARP pertencem a uma família de enzimas que catalisa a transferência de ADP-ribose a proteínas-alvo, utilizando dinucleótido de nicotinamida e adenina oxidado $\left(\mathrm{NAD}^{+}\right)$como substrato. Dessa forma, as PARP realizam importantes modificaçōes pós-traducionais. Já é descrita 
na literatura a relação das PARP com diversos mecanismos celulares, como reparo do DNA, estabilidade genômica e morte celular programada ${ }^{20-22}$.

A poli (ADP-ribose) polimerase-1 (PARP-1) localiza-se no núcleo das células, e é ativada em resposta a quebras de fitas simples no DNA. Uma vez ativada, a PARP-1 catalisa a transferência de ADP-ribose às proteínas-alvo, utilizando o $\mathrm{NAD}^{+}$como substrato, com a formação concomitante de nicotinamida. Como o $\mathrm{NAD}^{+}$é essencial para a reação de transporte de elétrons, foi demonstrado na literatura que a superativaçáo de PARP-1 pode conduzir a uma repressão da função mitocondrial, levando ao déficit de energia, à liberação do fator de indução de apoptose e, eventualmente, à morte celular ${ }^{21,23,24}$. Em células não proliferativas como cardiomiócitos, no entanto, o $\mathrm{NAD}^{+}$tem se mostrado compartimentalizado e não é facilmente esgotado pela ativaçáo PARP-1. Nas células não proliferativas citadas anteriormente, acredita-se que a superativação de PARP-1 ameace a sobrevivência da célula por inibir a atividade de outros caminhos $\mathrm{NAD}^{+}$ dependentes ${ }^{25,26}$. Vários fatores são grandemente afetados por mudanças nos níveis celulares de $\mathrm{NAD}^{+}-$as histonas desacetilases de classe III, também chamadas de sirtuínas ou proteínas SIRT, são um exemplo - que são homólogos ao gene Sir2 de leveduras, descritos no processo de silenciamento da cromatina, na sobrevivência da célula e no envelhecimento ${ }^{27,28}$.

O envolvimento de PARP-1 já foi descrito em processos que envolvem modulaçáo da resposta a danos ao DNA, no intuito de garantir a integridade genômica. Esses mecanismos são estritamente regulados e contribuem para a amplificação de sinais de danos no DNA, funcionando como uma espécie de interruptor entre o reparo de DNA ou induçáo da morte celular, o que justifica as constantes pesquisas realizadas com esses agentes terapêuticos no tratamento de vários tipos de cânceres ${ }^{29-33}$.É importante salientar também a interaçáo de letalidade sintética observada com a inibição PARP-1 e a deficiência em genes BRCA1 ou BRCA2, justificando os estudos em pacientes com mutaçóes germinativas em BRCA $^{12,22}$.

O primeiro ensaio aqui avaliado, utilizando veliparip, inibidor da PARP, demonstrou que a adição de carboplatina a um regime quimioterápico neoadjuvante padrão melhora a resposta clínica, embora os autores tenham reportado que dados essenciais dos estudos ainda estão em andamento, como biópsias e presença de biomarcadores dos diferentes grupos que seriam preditivos para definir melhor os subconjuntos de pacientes que mais se beneficiam com a adição de carboplatina em conjunto com veliparib ${ }^{11}$. No segundo ensaio utilizando inibidores da PARP, foi administrado o talazoparib, em pacientes com câncer de mama triplo-negativo, ressecável cirurgicamente, e portadores de mutação em BRCA, em que um único agente terapêutico, administrado uma vez ao dia, por via oral, no pré-operatório sem quimioterapia anterior ou durante o tratamento, produziu resultados clínicos significativos com toxicidade gerenciável ${ }^{12}$.

\section{CONCLUSÃO}

O tratamento do câncer de mama triplo-negativo, baseado no ponto de verificação imune, mudou radicalmente as abordagens terapêuticas para vários tipos de tumores, principalmente com a descoberta dos inibidores da PD-1 e do PD-L1.

Os inibidores da PD-1 e do PD-L1, quando combinados com outros agentes, parecem aumentar os benefícios tanto na resposta clínica quanto na taxa de sobrevivência e progressão da doença, principalmente nos casos de câncer metastático.

Além disso, o câncer de mama em estágio inicial parece ser ainda mais atraente do que o cenário metastático para a introdução dos inibidores da PD-1 e do PD-L1 tanto no cenário neoadjuvante quanto no adjuvante, uma vez que os tumores primários parecem mais imunogênicos do que os locais metastáticos.

Em relação à combinação de classes terapêuticas, a quimioterapia, por exemplo, pode induzir a múltiplas alteraçôes imunomodulatórias no microambiente tumoral, assim como outras modificaçóes podem influenciar positivamente a eficácia da imunoterapia e outras classes.

Em relação aos inibidores da PARP aqui citados, embora outros estudos randomizados em pacientes com câncer de mama triplo-negativo tenham demonstrado que a adição de carboplatina, com ou sem inibidores da poli PARP, à quimioterapia neoadjuvante aumenta a probabilidade de se obter uma resposta patológica completa, o uso dessas terapias nesse cenário permaneceu controverso. $\mathrm{Na}$ avaliaçáo de inibidores da PARP em pacientes com tumores ressecáveis cirurgicamente e que não haviam utilizado nenhuma classe de quimioterápicos anteriormente ou durante o ensaio, já se espera uma melhor taxa de resolutividade desses tumores, diferentemente dos tumores avançados e metastáticos descritos em outros estudos. Os pacientes devem ser estratificados de acordo com biomarcadores específicos.

Os diversos estudos em andamento podem lançar luz sobre os biomarcadores da resposta imune ao câncer de mama triplo-negativo e ajudar a determinar se um conjunto de características tumorais, como coloraçôes por imuno-histoquímica, pode prever uma eficácia melhor na utilização da imunoterapia. 


\section{CONTRIBUIÇÕES}

Dione Fernandes Tavares e Renata Lopes Britto contribuíram na concepção e/ou no planejamento do estudo; na obtenção, análise e interpretação dos dados; assim como na redação e revisão crítica com contribuição intelectual. Laércio Moreira Cardoso-Júnior e Victoria Chaves Ribeiro participaram da redação e revisão crítica com contribuição intelectual. Todos os autores aprovaram a versão final a ser publicada.

\section{DECLARAÇÃO DE CONFLITO DE INTERESSES}

Nada a declarar.

\section{FONTES DE FINANCIAMENTO}

Coordenação de Aperfeiçoamento de Pessoal de Nível Superior (Capes), Brasil - Código de Financiamento 001.

\section{REFERÊNCIAS}

1. Perou CM, Sørlie T, Eisen MB, et al. Molecular portraits of human breast tumours. Nature. 2000;406(6797):74752. doi: http://doi.org/10.1038/35021093

2. Sørlie T, Perou CM, Tibshirani R, et al. Gene expression patterns of breast carcinomas distinguish tumor subclasses with clinical implications. Proc Natl Acad Sci U S A. 2001;98(19):10869-74. doi: http://doi. org/10.1073/pnas. 191367098

3. Instituto Nacional de Câncer José Alencar Gomes da Silva. Estimativa 2020: incidência de câncer no Brasil [Internet]. Rio de Janeiro: INCA; 2019 [acesso 2020 ago 22]. Disponível em: https://www.inca.gov.br/publicacoes/ livros/estimativa-2020-incidencia-de-cancer-no-brasil

4. Shah TA, Guraya SS. Breast cancer screening programs: review of merits, demerits, and recent recommendations practiced across the world. J Microsc Ultrastruct. 2017;5(2):59-69. doi: http://doi.org/10.1016/j. jmau.2016.10.002

5. Schmid P, Adams S, Rugo HS, et al. Atezolizumab and Nab-paclitaxel in advanced triple-negative breast cancer. N Engl J Med. 2018;379(22):2108-21. doi: http://doi. org/10.1056/NEJMoa1809615

6. Nanda R, Chow LQM, Dees EC, et al. Pembrolizumab in patients with advanced triple-negative breast cancer: phase Ib KEYNOTE-012 study. J Clin Oncol. 2016;34(21):2460-7. doi: http://doi.org/10.1200/ JCO.2015.64.8931

7. Adams S, Schmid P, Rugo HS, et al. Pembrolizumab monotherapy for previously treated metastatic triplenegative breast cancer: cohort A of the phase II KEYNOTE-086 study. Ann Oncol. 2019;30(3):397404. doi: http://doi.org/10.1093/annonc/mdy517
8. Tolaney S, Kalinsky K, Kaklamani V, et al. Abstract PD6-13: phase $1 \mathrm{~b} / 2$ study to evaluate eribulin mesylate in combination with Pembrolizumab in patients with metastatic triple-negative breast cancer. Cancer Res. 2018;78(4 Suppl):PD6-13. doi: https://doi.org/ 10.1158/1538-7445.SABCS17-PD6-13

9. Dirix LY, Takacs I, Jerusalem G, et al. Avelumab, an anti-PD-L1 antibody, in patients with locally advanced or metastatic breast cancer: a phase 1b JAVELIN solid tumor study. Breast Cancer Res Treat. 2018;167(3):67186. doi: http://doi.org/10.1007/s10549-017-4537-5

10. Emens LA, Cruz C, Eder JP, et al. Long-term clinical outcomes and biomarker analyses of Atezolizumab therapy for patients with metastatic triple-negative breast cancer: a phase 1 study. JAMA Oncol. 2019;5(1):74-82. doi: http://doi.org/10.1001/jamaoncol.2018.4224

11. Loibl S, O'Shaughnessy J, Untch M, et al. Addition of the PARP inhibitor veliparib plus carboplatin or carboplatin alone to standard neoadjuvant chemotherapy in triplenegative breast cancer (BrighTNess): a randomised, phase 3 trial. Lancet Oncol. 2018;19(4):497-509. doi: http:// doi.org/10.1016/S1470-2045(18)30111-6

12. Litton JK, Scoggins M, Hess KR, et al. Neoadjuvant talazoparib (TALA) for operable breast cancer patients with a BRCA mutation (BRCA+). J Clin Oncol. 2018;36(Suppl 15):508. doi: http://doi.org/10.1200/ JCO.2018.36.15_suppl.508

13. Souza MT, Silva MD, Carvalho R. Revisão integrativa: o que é e como fazer. Einstein (São Paulo). 2010;8(1):1026. doi: https://doi.org/10.1590/s1679-45082010rw1134

14. Ribas A, Hu-Lieskovan S. What does PD-L1 positive or negative mean? J Exp Med. 2016;213(13):2835-40. doi: http://doi.org/10.1084/jem.20161462

15. Kythreotou A, Siddique A, Mauri FA, et al. PD-L1. J Clin Pathol. 2018;71(3):189-94. doi: http://doi.org/10.1136/ jclinpath-2017-204853

16. Pol J, Vacchelli E, Aranda F, et al. Trial watch: immunogenic cell death inducers for anticancer chemotherapy. Oncoimmunology. 2015;4(4):e1008866. doi: http://doi.org/10.1080/2162402X.2015.1008866

17. Heinhuis KM, Ros W, Kok M, et al. Enhancing antitumor response by combining immune checkpoint inhibitors with chemotherapy in solid tumors. Ann Oncol. 2019;30(2):219-35. doi: http://doi.org/10.1093/ annonc/mdy551

18. Galluzzi L, Buqué A, Kepp O, et al. Immunogenic cell death in cancer and infectious disease. Nat Rev Immunol. 2017;17(2):97-111. doi: http://doi.org/10.1038/ nri.2016.107

19. Roselli M, Cereda V, di Bari MG, et al. Effects of conventional therapeutic interventions on the number and function of regulatory $\mathrm{T}$ cells. Oncoimmunology. 2013;2(10):e27025. doi: http://doi.org/10.4161/ onci. 27025 
20. Ghiringhelli F, Menard C, Puig PE, et al. Metronomic cyclophosphamide regimen selectively depletes $\mathrm{CD} 4{ }^{+} \mathrm{CD} 25^{+}$regulatory $\mathrm{T}$ cells and restores $\mathrm{T}$ and $\mathrm{NK}$ effector functions in end stage cancer patients. Cancer Immunol Immunother. 2007;56(5):641-8. doi: http:// doi.org/10.1007/s00262-006-0225-8

21. Leung AKL. Poly(ADP-ribose): an organizer of cellular architecture. J Cell Biol. 2014;205(5):613-9. doi: https:// doi.org/10.1083/jcb.201402114

22. Sousa FG, Matuo R, Soares DG et al. PARPs and the DNA damage response. Carcinogenesis. 2012;33(8):1433-40. doi: https://doi.org/10.1093/carcin/bgs132

23. Bürkle A. Physiology and pathophysiology of poly(ADPribosyl)ation. Bioessays. 2001;23(9):795-806. doi: https://doi.org/10.1002/bies.1115

24. Bürkle A. PARP-1: a regulator of genomic stability linked with mammalian longevity. Chembiochem. 2001;2(10):725-8. doi: https://doi.org/10.1002/14397633(20011001)2:10<725::AID-CBIC725>3.0.CO;2-3

25. Virág L, Szabó C. The therapeutic potential of poly(ADP-ribose) polymerase inhibitors. Pharmacol Rev. 2002;54(3):375-429. doi: https://doi.org/10.1124/ pr.54.3.375

26. Chiarugi A. Poly(ADP-ribose) polymerase: killer or conspirator? The 'suicide hypothesis' revisited. Trends Pharmacol Sci. 2002;23(3):122-9. doi: https://doi. org/10.1016/S0165-6147(00)01902-7

27. Ying W, Alano CC, Garnier P, et al. NAD+ as a metabolic link between DNA damage and cell death. J Neurosci Res. 2005;79(1-2):216-23. doi: https://doi.org/10.1002/ jnr.20289

28. Imai S, Armstrong CM, Kaeberlein M, et al. Transcriptional silencing and longevity protein Sir 2 is an NAD-dependent histone deacetylase. Nature. 2000;403(6771):795-800. doi: https://doi.org/10.1038/35001622

29. North BJ, Verdin E. Sirtuins: Sir2-related NAD-dependent protein deacetylases. Genome Biol. 2004;5(5):224. doi: https://doi.org/10.1186/gb-2004-5-5-224

30. Mortusewicz O, Amé JC, Schreiber V, et al. Feedbackregulated poly(ADP-ribosyl)ation by PARP-1 is required for rapid response to DNA damage in living cells. Nucleic Acids Res. 2007;35(22):7665-75. doi: https:// doi.org/10.1093/nar/gkm933

31. Vodenicharov MD, Ghodgaonkar MM, Halappanavar SS, et al. Mechanism of early biphasic activation of poly(ADP-ribose) polymerase-1 in response to ultraviolet B radiation. J Cell Sci. 2005;118(Pt 3):589-99. doi: https://doi.org/10.1242/jcs.01636

32. Damiani RM, Moura DJ, Viau CM. et al. Pathways of cardiac toxicity: comparison between chemotherapeutic drugs doxorubicin and mitoxantrone. Arch Toxicol. 2016;90(9):2063-76. doi: https://doi.org/10.1007/ s00204-016-1759-y
33. Damiani RM, Moura DJ, Viau CM, et al. Influence of PARP-1 inhibition in the cardiotoxicity of the topoisomerase 2 inhibitors doxorubicin and mitoxantrone. Toxicol In Vitro. 2018;52:203-13. doi: https://doi.org/10.1016/j.tiv.2018.06.013

Recebido em 8/5/2020 Aprovado em 10/11/2020 